\title{
Selective excitation luminescence in bulk-grown GaAs
}

A. T. Hunter, and T. C. McGill

Citation: Appl. Phys. Lett. 40, 169 (1982); doi: 10.1063/1.93031

View online: https://doi.org/10.1063/1.93031

View Table of Contents: http://aip.scitation.org/toc/apl/40/2

Published by the American Institute of Physics

\section{Conference Proceedings}

Get $30 \%$ off all

Enter Promotion Code PDF-30 at checkout print proceedings! 


\title{
Selective excitation luminescence in bulk-grown GaAs
}

\author{
A. T. Hunter and T. C. McGill \\ California Institute of Technology, Pasadena, California 91125
}

(Received 6 July 1981; accepted for publication 2 October 1981)

\begin{abstract}
We have measured the excited state levels of two different shallow acceptors in bulk-grown GaAs, using selective excitation luminescence. The $1 S-2 S$ energy differences were measured to be 21.5 and $18.5 \mathrm{meV}$, respectively. By comparing these values to those measured by two-hole transition luminescence in high quality epitaxial GaAs [Ashen et al., J. Phys. Chem. Solids 36, 1041 (1975)], the acceptors were identified as $\mathrm{Zn}$ and $\mathrm{C}$. The measured $1 S-2 P$ energy differences also support the identification. These studies demonstrate that selective excitation luminescence can be used to identify shallow acceptors in bulk-grown semi-insulating GaAs, and hence can be used as a diagnostic tool for bulk-grown samples.
\end{abstract}

PACS numbers: 78.55.Ds, 71.55.Fr, 78.60. - b

The identification of shallow acceptors in GaAs by the use of straightforward photoluminescence is fairly well advanced for high quality epitaxial samples. 'However, there are additional problems in bulk-grown GaAs such as high defect concentrations and low luminescence intensity that limit the usefulness of these methods. Selective excitation luminescence, ${ }^{2-5}$ in which a tunable, below band-gap laser is used to selectively excite the shallow impurities of interest, seems to overcome the problems in identifying these impurities in bulk-grown GaAs. This technique has been used successfully in bulk-grown InP, ${ }^{2,3}$ and also has been used in $\mathrm{ZnTe}$ (Ref. 2) and $\mathrm{ZnSe}$. ${ }^{4.5}$

In this letter, we report for the first time results for GaAs. We were able to conclusively identify the principal acceptor in two bulk-grown samples, neither of which were intentionally doped with shallow acceptors. Both samples studied were grown by the liquid encapsulated Czochralski method. ${ }^{6}$ Sample 1 was chrome doped, and is semi-insulating. ${ }^{6}$ Sample 2 was not intentionally doped, and is $p$ type with $N_{A}-N_{D} \sim 10^{16} \mathrm{~cm}^{-3}$. 6 We have identified the accep-

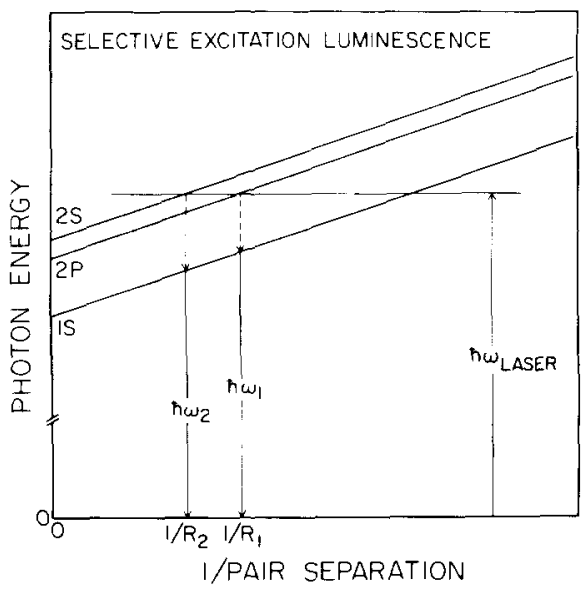

FIG. 1. Schematic of the selective excitation luminescence process. ${ }^{2,3}$ Energy required to create a donor-acceptor pair is plotted against the inverse to the pair separation. The dye laser creates pairs with the hole in an excited state. The hole quickly relaxes to the $1 S$ ground state, then radiatively recombines with the electron. The difference between the energy of the laser and that of the emitted light provides the ground-state-excited state energy difference. tor as $\mathrm{Zn}$ for the first sample, and $\mathrm{C}$ for the second, as will be shown below.

The experiment is essentially that used for $\mathrm{ZnSe}$ in Refs. 4 and 5. A tunable laser is used to create an electron on a donor and a hole on an adjacent acceptor, with the hole in an excited state. In principle, pairs should be created with donor electrons in excited states as well, but lines due to this process are not observed. The first excited state of the donor is only $\sim 1.5 \mathrm{meV}$ from the band edge, and these weakly bound states may be thermally ionized with high probability. Also, the very extended wavefunction of the excited donor may be substantially broadened by interaction with other defects. This could lead to a line breadth which is too wide to resolve against background luminescence. The energy required to create the pair with an excited hole is $\hbar \omega_{\text {laser }}=E_{g}$ $-E_{A}^{*}-E_{D}+e^{2} / \epsilon R+J^{*}(R)$, where $E_{g}$ is the band gap, $E_{A}^{*}$ the energy of the excited hole with respect to the valence band, $E_{D}$ the energy of the electron with respect to the conduction band, $e^{2} / \epsilon R$ is the Coulomb attraction of the ionized centers at distance $R$ from one another, and $J *(R)$ represents the interaction of the donor and excited acceptor wave functions. This is illustrated in Fig. 1, where the energy of the occupied pair is plotted against the inverse of the pair separation with $J^{*}(R)$ taken to be zero. The laser will create pairs with the hole in the first excited state at pair separation $R_{1}$, and holes in the second excited state at separation $R_{2}$. The holes very quickly relax to their respective ground state, then recombine with the electrons, emitting light at $\hbar \omega=E_{g}-E_{A}-E_{D}+e^{2} / \epsilon R+J(R)$, where $E_{A}$ is now the energy of the ground state of the hole with respect to the valence band, and $J(R)$ is the interaction between the donor and the ground state acceptor wave function. The energy difference between the laser and emitted light consists of two terms in this experiment: $\Delta E=\left(E_{A}-E_{A}^{*}\right)+\left[J^{*}(R)\right.$ $-J(R)]$. Both $J(R)$ and $J^{*}(R)$ gotozerofor sufficiently separated pairs, and in this limit $\Delta E$ is just the difference between the ground and excited state energy of the acceptor wave functions.

The equipment and experimental procedure used are as follows. A Coherent CR-3000K ion laser was used to pump a Coherent model 590 dye laser, with a carbo-cyanine dye. The dye laser was tuned with a 3-plate birefringent filter 
placed in the dye-laser cavity. This tuning element allowed linewidths of between 0.1 and $0.2 \mathrm{meV}$, which allowed energy resolution of better than $0.1 \mathrm{meV}$ in most cases, because the line shapes were symmetric Gaussians, the peaks of which could be determined with high accuracy. The wavelength of light used for the experiments was such that the ground-state emission was near the peak of the donor-acceptor band. The laser light was then focused onto a sample, which was immersed in liquid helium pumped below the lambda point in a Janis cryogenic dewar. The emitted light was analyzed by scanning a Spex 1404 double grating spectrometer and detected with a GaAs photomultiplier tube.

The gratings used were replica gratings, but ghost and background intensity proved to be insignificant at the wavelength differences of interest in this experiment. Luminescence background was not insignificant, but could be easily distinguished from the scattering processes we wished to observe. This was done by taking additional spectra with the pump laser at slightly different wavelengths. Those features described above shifted in energy with the laser, while background luminescence did not.

Figure 2 shows luminescence spectra for the two bulkgrown GaAs samples used in this study. In the upper spectrum, the signal-to-noise ratio is not large enough to resolve the bound-exciton line. In the bottom spectrum, it can just barely be resolved on an expanded scale at $1512 \mathrm{meV}$. The two-hole transition ${ }^{7.8}$ of the bound-exciton line (which leaves the remaining hole in an excited state) cannot be resolved at all. In principle, the donor-acceptor pair luminescence bands centered at $1490 \mathrm{meV}$ can be used to identify the acceptor involved in the transition. ${ }^{1}$ However, this line has a rather high intrinsic width, as does the free-electron to bound-hold band seen at higher temperatures. It is difficult to differentiate between acceptors with ionization energies that differ by only a few milli-electron volts, especially when the signal-to-noise ratio is as poor as it is in the upper spectrum. Even with sufficiently good signal-to-noise, line shifts due to high impurity concentrations, which are quite possible in bulk-grown material, make identification by the position of the donor-acceptor band questionable. ${ }^{1}$

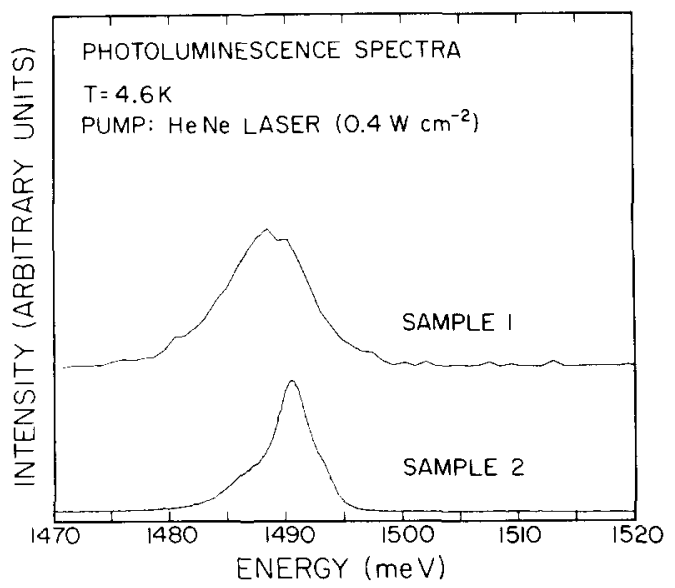

FIG. 2. Photoluminescence spectra for two samples of bulk-grown GaAs, with above band-gap excitation. Intensity of emitted light is plotted on the vertical scale, against photon energy on the horizontal scale.
Selective excitation luminescence data, however, provides an unambiguous identification of the acceptors. The top three spectra in Fig. 3 are from sample 1, each taken with the dye laser at different photon energy. The spectra show intensity of luminescence plotted against energy difference between the laser and luminescence. This means the zero of energy in each case is the laser energy indicated above the spectrum at the right. Four peaks are apparent which remain at an almost constant energy from the laser. The most intense peak is located $\sim 21.5 \mathrm{meV}$ below the laser line, and is seen in all three spectra. The line does shift slightly as the energy of the pump laser is changed, which was determined by carefully measuring the energy differences for the spectra shown here, as well as for other spectra. This shift is due to the interaction between the donor and acceptor wave functions described earlier, and causes the energy difference to decrease as the pair separation is decreased. The shift is less than $0.1 \mathrm{meV}$ (which is the uncertainty of the energy separation measurement for this sample) for pump laser energies of $1509.7 \mathrm{meV}$ and smaller. This pump energy creates pairs with approximately 200 - $\AA$ pair separation. At a pump energy of $1512.6 \mathrm{meV}$, corresponding to $\sim 130$ - $\AA$ pair separations, the energy shift is $0.5 \mathrm{meV}$. In addition to this fairly intense peak, three other less intense peaks can be resolved, though not in all the spectra. They occur at 19.2,21.5, and $25.0 \mathrm{meV}$. Interpreting the data as was done in Refs. 2 and 3, the principal line is assigned to the $1 S-2 S$ energy difference, and the less intense lines are assigned to the $1 S-2 P$ differences.

These results can now be compared to theoretical calculations, ${ }^{9}$ and to measurements made on epitaxial GaAs by other methods. These earlier results, and the results of this study, are tabulated in Table I. The two-hole shift measured for $\mathrm{Zn}$-doped epitaxial samples is $21.8 \mathrm{meV}^{1,8}$ and is very close to the $21.5-\mathrm{meV} 1 S-2 S$ difference measured for sample 1. The position of the less intense peaks line up well with the

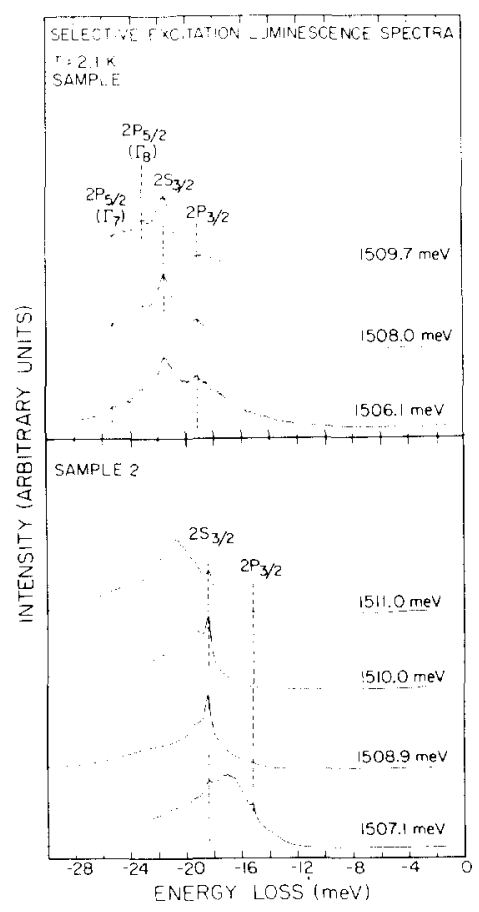

FIG. 3. Selective excitation luminescence spectra for two samples of bulk-grown GaAs. Intensity of emitted light is plotted against the difference in photon energy of emitted light and that of the laser. Photon energy of the laser for each spectrum is given over the spectrum at the right. The top three spectra are for sample 1 , the bottom four for sample 2 . The labels for the acceptor lev els are those used in Ref. 9. 
TABLE I. Ground state to excited state splittings for the shallow acceptors $\mathrm{C}$ and $\mathrm{Zn}$ in GaAs. The labels for the acceptor levels are those used in Ref. 9.

\begin{tabular}{|c|c|c|c|c|}
\hline & $\begin{array}{l}1 S_{3 / 2}-2 P_{3 / 2} \\
(\mathrm{meV})\end{array}$ & $\begin{array}{l}1 S_{3 / 2}-2 S_{3 / 2} \\
(\mathrm{meV})\end{array}$ & $\begin{array}{l}1 S_{3 / 2}-2 P_{5 / 2}\left(\Gamma_{\mathrm{g}}\right) \\
(\mathrm{meV})\end{array}$ & $\begin{array}{l}1 S_{3 / 2}-2 P_{5 / 2}\left(\Gamma_{7}\right) \\
(\mathrm{meV})\end{array}$ \\
\hline \multicolumn{5}{|l|}{$\mathrm{Zn}$} \\
\hline Theory ${ }^{a}$ & 19.3 & 23.1 & 23.5 & 25.4 \\
\hline Photoconductivity ${ }^{b}$ & 19.4 & $\ldots$ & 23.2 & 25.0 \\
\hline "Two-hole" shift ${ }^{c}$ & $\cdots$ & 21.8 & $\cdots$ & $\cdots$ \\
\hline This work & 19.2 & 21.5 & 23.1 & 25.0 \\
\hline \multicolumn{5}{|l|}{$\mathrm{C}$} \\
\hline Theory ${ }^{a}$ & 14.6 & 18.4 & 18.8 & 20.7 \\
\hline Photoconductivity ${ }^{b}$ & 15.2 & $\cdots$ & 19.3 & 21.3 \\
\hline "Two-hole" shift" & $\cdots$ & 18.5 & $\cdots$ & $\ldots$ \\
\hline This work & 15.1 & 18.5 & $\ldots$ & $\cdots$ \\
\hline
\end{tabular}

${ }^{a}$ Deduced from theoretical values quoted in Ref. 9 by replacing the calculated $(S$ position $(25.7$ meV) with that measured in Ref. $\mathrm{l}: 26.0$ meV for $\mathrm{C}$ and 30.7 meV for $\mathrm{Zn}$.

${ }^{b}$ Measured on epitaxial GaAs from Ref. 10.

Measured on epitaxial GaAs from Ref. 1.

$1 S-2 P$ differences measured using photoconductivity ${ }^{10}$ on epitaxial GaAs. Also included in the table, for reference, are the energy differences deduced from the effective mass calculation of Baldereschi and Lipari, ${ }^{9}$ by replacing the calculated $1 S$ position with that measured by Ashen et al. ${ }^{1}$ The matchup between our results and both theory and earlier results indicate that the acceptor in this sample is $\mathrm{Zn}$. It also demonstrates that selective excitation luminescence provides an unambiguous method of determining the identity of shallow acceptors in bulk-grown semi-insulating GaAs.

We have also used this method to identify the acceptor in the second sample as carbon. The bottom half of Fig. 3 shows four spectra taken on this sample, each at a different laser energy. Only two peaks are visible on these spectra, but they are sufficient to identify the acceptor. The principal line in this case is $18.5 \mathrm{meV}$ below the laser energy (and is somewhat narrower than the principal line in sample 1) The 18.5meV difference is again the energy difference extrapolated to the distant pair limit, which was determined by making the measurement for a wide range of pump energies. The 18.5$\mathrm{meV}$ value is the two-hole shift measured for carbon-doped epitaxial samples, ${ }^{1,8}$ and identifies the dopant in our sample as carbon. The second line, at $15.2 \mathrm{meV}$, confirms the identification, because this is the $1 S-2 P_{3 / 2}$ shift measured for carbon using photoconductivity. ${ }^{10}$ These results are also tabulated in Table $\mathbf{I}$.

Two points concerning line breadth and height could use further discussion. The increased linewidth in sample 1 could be indicative of much higher ionized impurity concentration. The electric fields of nearly charged impurities can inhomogeneously broaden the impurity levels through a second-order Stark shift. ${ }^{11}$ In addition to shifting the impurity energy levels, the Stark effect also mixes $S$ and $P$ wavefunctions. Since the hole must undergo what is apparently a parity forbidden relaxation process ${ }^{2}$ to reach the ground state from a $P$-like excited state, this mixing would enhance the relaxation from the $2 P$ excited states to the $1 S$ ground state.
Therefore, both the breadth of the lines and the increased intensity of the $1 S-2 P$ lines in sample 1 could be explained by a much higher ionized impurity concentration in this sample than in sample 2 .

In conclusion, we have measured the excited-state levels of shallow acceptors in bulk-grown $\mathrm{GaAs}$, using selective excitation luminescence. While these levels have been measured in GaAs before, it was done using different methods in high quality, epitaxial layers. This is the first time the levels have been measured in bulk-grown GaAs, where the other methods are not as useful. In doing so, selective excitation luminescence has been shown to be useful in identifying the shallow acceptors in bulk-grown GaAs.

We would like to acknowledge the support from the Office of Naval Research under Contract No. N00014-81K-0305. The authors also gratefully acknowledge H. Kimura, O. J. Marsh, and H. V. Winston of Hughes Research Laboratories for providing the samples and the results of Hall-effect measurements on the samples.

'D. J. Ashen, P. J. Dean, D. T. J. Hurle, J. B. Mullin, A. M. White, and P. D. Greene, J. Phys. Chem. Solids 36, 1041 (1975).

${ }^{2}$ P. J. Dean, D. J. Robbins, and S. G. Bishop, Solid State Commun. 32, 379 (1979).

${ }^{3}$ P. J. Dean, D. J. Robbins, and S. G. Bishop, J. Phys. C 12, 5567 (1979).

${ }^{4} H$. Tews and $H$. Venghaus, Solid State Commun. 30, 219 (1979).

${ }^{5}$ H. Tews, H. Venghaus, and P. J. Dean, Phys. Rev. B 19, 5178 (1979).

${ }^{\circ} \mathrm{H}$. V. Winston (private communication).

'W. Schairer and T. O. Yep, Solid State Commun. 9, 421 (1971).

${ }^{\text {B} A . ~ M . ~ W h i t e, ~ P . ~ J . ~ D e a n, ~ D . ~ J . ~ A s h e n, ~ J . ~ B . ~ M u l l i n, ~ M . ~ W e b b, ~ B . ~ D a y, ~ a n d ~}$ P. D. Greene, J. Phys. C 6, L 243 (1973).

${ }^{9}$ A. Baldereschi and N. O. Lipari, Phys. Rev. B 9, 1525 (1974).

${ }^{10}$ R. F. Kirkman, R. A. Stradling, and P. J. Lin-Chung, J. Phys. C 11, 419 (1978).

'D. M. Larsen, Phys. Rev. B 8, 535 (1973). 